\title{
A Parametric study of AC electric field-induced toroidal vortex formation in laminar nonpremixed coflow flames
}

\author{
Yuan Xiong, Suk Ho Chung, Min Suk Cha* \\ King Abdullah University of Science and Technology (KAUST) \\ Clean Combustion Research Center (CCRC) \\ Thuwal, Saudi Arabia
}

\begin{abstract}
This study presents an experimental work investigating the controlling parameters on the formation of an electrically-induced inner toroidal vortex (ITV) near a nozzle rim in small, laminar nonpremixed coflow flames, when an alternating current is applied to the nozzle. A systematic parametric study was conducted by varying the flow parameters of the fuel and coflowing-air velocities, and the nozzle diameter. The fuels tested were methane, ethylene, ethane, propane, n-butane, and i-butane, each representing different ion-generation characteristics and sooting tendencies. The results showed that the fluid dynamic effects on ITV formation were weak, causing only mild variation when altering flow velocities. However, increased fuel velocity resulted in increased polycyclic aromatic hydrocarbon (PAH) formation, which promoted ITV formation. When judging the ITV-formation tendency based on critical applied voltage and frequency, it was qualitatively well correlated with the PAH concentration and the relative location of PAHs to the nozzle rim. The sooting tendency of the fuels did not affect the results much. A change in the nozzle diameter highlighted the importance of the relative distance between the PAH zone and the nozzle rim, indicating the role of local electric-field intensity on ITV formation. Detailed onset conditions, characteristics of near-nozzle flow patterns, and PAH distributions are also discussed.
\end{abstract}

Keywords: electric field, nonpremixed flame, toroidal vortex, polycyclic aromatic hydrocarbons, ionic wind. 


\section{Introduction}

An electric field was recently proposed as an alternative method to modulate and control various combustion aspects, including flame propagation [1-7], flow structure [4-9], and critical phenomena such as liftoff and blowoff [10-13]. Various mechanisms based on ionic wind [14], modified chemistry [15] and transport property [16], and thermal effects due to ohmic heating [17] have been proposed to explain the observed phenomena, while recent investigations of nonpremixed edge flames $[6,7]$ found that modified flow fields caused by ionic wind can be a major effect of electric fields.

Many previous studies have shown that charged-species motion under electric fields (Lorentz force) generates a bulk-flow motion (ionic wind), resulting in a significant modification of the flow fields [5-10, 18-20]. Utilizing the ionic wind under a sub-critical electric field, which is lower than a breakdown field for electrical discharge, to control flame characteristics may have a few advantages. First, integrating electric fields into combustors would be simple since most fuel injection nozzles are good conductors. Second, a sub-critical electric field typically consumes less electrical power than the thermal power of flames, by several orders of magnitude. Third, the response time to establish ionic winds is typically $\mathrm{O}(\mathrm{ms})$, which is feasible to implement in a feedback control system.

Recent investigation [5] into a small propane coflow diffusion flame showed that applying alternating current $(\mathrm{AC})$ to a jet nozzle could trigger the formation of a recirculation zone in a fuel core near the nozzle exit. The recirculation zone was identified as an inner toroidal vortex (ITV) pairing with an outer toroidal vortex (OTV). The sharp boundary of the critical moment of ITV formation in terms of $V_{\text {ac }}$ and $f_{\text {ac }}$ was reported [5], indicating a potential control of the diffusion flames by modulating a flow 
pattern with AC. The formation of the ITV influenced the distributions of polycyclic aromatic hydrocarbons (PAHs) and soot particles. Hence, a possible mechanism for ITV formation was considered, as the result of ionic wind and potential interaction with AC-induced magnetic fields. However, the detailed mechanism is not yet clear and, even for a hypothesized mechanism, the major source of responsible ions for an ionic windgenerating ITV needs to be further clarified. Note that numerical approaches have been performed particularly in one-dimensional configurations trying to validate detailed ion chemistry and electric current response to external bias voltage [21-23], which, however, could not be applicable to a prediction of modified flow field due to their intrinsic one-dimensional nature. To expand present efforts in numerical simulations to multi-dimensional one, identification of charged species and their evolution (e.g., electron attachment) in a flow volume should be correctly implemented to capture a flow modification resulted from electric body force.

Flames are known to produce various charged species. For a fuel-lean, or near stoichiometric premixed hydrocarbon flames, a chemi-ionization reaction, $\mathrm{CH}+\mathrm{O} \rightarrow$ $\mathrm{CHO}^{+}+\mathrm{e}$, is the initiation step for charge generation [24], and the subsequent proton transfer reaction, $\mathrm{CHO}^{+}+\mathrm{H}_{2} \mathrm{O} \rightarrow \mathrm{H}_{3} \mathrm{O}^{+}+\mathrm{CO}$, results in a relatively large amount of hydronium ions in a reaction zone and also in a post-combustion region $[25,26]$. While in fuel-rich flames, $\mathrm{C}_{3} \mathrm{H}_{3}{ }^{+}$becomes a dominant ion near the reaction zone, and a peak ion concentration can be found downstream of the one-dimensional rich premixed flames, where soot inception occurs [27], due to ionized PAHs and charged nascent soot particles. Soot particles typically become charged via a thermal-ionization process, while ionized PAHs can possibly form from either $\mathrm{C}_{3} \mathrm{H}_{3}{ }^{+}$or from additional chemi-ionization processes 
involving neutral PAHs near the sooting zone [28]. Although there are no detailed studies on ions in a diffusion flame, all aforementioned charged species may be important, since a diffusion flame has a wide range of local mixture fraction in a reaction zone.

The objective of the present study is to provide further experimental data for the future development of a model of ITV formation. In this regard, the effects of various parameters on ITV formation in a small jet diffusion flame with AC electric fields were explored. Those parameters included the jet velocity (flow parameter), the nozzle diameter (geometrical parameter), and the fuel type (chemical parameter, which has a different tendency for the formation of PAHs and soot) to better understand the origin of ITV formation.

\section{Experiment}

The apparatus, similar to the one reported previously [5], consisted of a coflow burner, flow controllers, an AC power supply unit, a voltage measurement setup, and laser diagnostic setups to visualize PAHs and flow fields. A schematic of the coflow burner and the electrical configuration is depicted in Fig. 1. A fuel nozzle, having an inner diameter $d$, protruded $8.7 \mathrm{~mm}$ above the exit of the coflowing air. The nozzle length was designed to have a fully developed velocity profile at the exit in an ideal condition, although a velocity profile at the fuel nozzle exit is significantly altered depending on fuel density in a small laminar coflow diffusion flame [29]. The size of the coflow section was $150 \mathrm{~mm}$ in diameter and air was supplied through both the layers of beads and a ceramic honeycomb, for a uniform and laminar flow. The flow rates of the fuel and coflow air streams were monitored by mass flow controllers. To apply a high voltage to 
the fuel nozzle, the coflow body was made of non-conducting acetal resin. A circular, stainless steel mesh $(15 \mathrm{~cm}$ in diameter) was positioned $27 \mathrm{~cm}$ above the nozzle tip, serving as a ground electrode.

Detailed experimental conditions are summarized in Table 1. The baseline condition was a propane jet through a nozzle of $d=10.8 \mathrm{~mm}$, with a fuel jet velocity $\left(U_{\mathrm{f}}\right)$ of $1.4 \mathrm{~cm} / \mathrm{s}$, and a coflow air velocity $\left(U_{\mathrm{c}}\right)$ of $6.2 \mathrm{~cm} / \mathrm{s}$. When the effects of the flow velocities were tested, the fuel and coflow air velocities were varied from the baseline condition. The effect of the fuel was tested by varying the fuel type (methane, ethylene, ethane, n-butane, and i-butane), and the effect of the nozzle diameter was also tested. For tests of the effects of fuel and nozzle diameter, the fuel jet velocity was adjusted so that the visible flame height, determined by its yellow luminosity, was similar to that of the baseline flame, which will be detailed in a corresponding section.

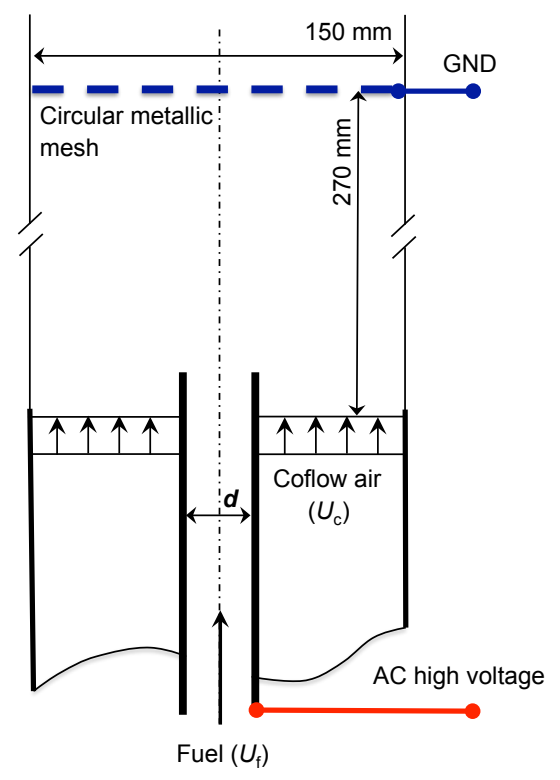

Fig.1. Schematic of experimental setup.

Table 1. Experimental conditions (boldface indicates baseline condition) 


\begin{tabular}{|c|c|c|c|c|c|c|c|c|c|c|c|}
\hline \multirow{4}{*}{$\begin{array}{c}\text { Nozzle I.D. } \\
d[\mathrm{~mm}] \\
\text { Fuel }\end{array}$} & \multicolumn{11}{|c|}{ Experimental parameters varied } \\
\hline & \multirow{2}{*}{$\begin{array}{c}\text { Jet Velocity } \\
10.8\end{array}$} & \multicolumn{5}{|c|}{ Fuel } & \multicolumn{5}{|c|}{ Nozzle diameter } \\
\hline & & \multicolumn{5}{|c|}{10.8} & 2.8 & 4.5 & 7.6 & 10.8 & 12.5 \\
\hline & $\mathrm{C}_{3} \mathrm{H}_{8}$ & $\mathrm{C}_{2} \mathrm{H}_{4}$ & $\mathrm{C}_{2} \mathrm{H}_{6}$ & $\mathrm{C}_{3} \mathrm{H}_{8}$ & $\mathrm{n}-\mathrm{C}_{4} \mathrm{H}_{10}$ & $\mathrm{i}-\mathrm{C}_{4} \mathrm{H}_{10}$ & & & $\mathrm{C}_{3} \mathrm{H}_{8}$ & & \\
\hline $\begin{array}{c}\text { Fuel velocity } \\
U_{\mathrm{f}}[\mathrm{cm} / \mathrm{s}]\end{array}$ & $0.71 / 1.4 / 2.1 / 2.8$ & 2.2 & 2.2 & 1.4 & 1.0 & 0.9 & 18.3 & 7.5 & 2.7 & 1.4 & 1 \\
\hline $\begin{array}{c}\text { Coflow velocity } \\
U_{\mathrm{c}}[\mathrm{cm} / \mathrm{s}]\end{array}$ & $2.1 / 6.2 / 8.3$ & \multicolumn{5}{|c|}{6.2} & \multicolumn{5}{|c|}{6.2} \\
\hline
\end{tabular}

The AC power supply unit was composed of a power amplifier (TREK, 30/20A) and a function generator, which generated a sinusoidal-signal input to the amplifier. The tested applied voltage $\left(V_{\text {ac }}\right)$ and frequency $\left(f_{\text {ac }}\right)$ to the fuel nozzle were $0-5 \mathrm{kV}(\mathrm{rms})$ and 0-5 kHz, respectively. In all cases, the voltage was applied after a 10 -minute operation with a flame, so that the burner system could reach a thermal-equilibrium state [29]. After each test, the fuel nozzle was carefully cleaned to remove deposited soot and to avoid any consequent uncertainty.

PAHs were measured using a planar laser-induced fluorescence (PLIF) technique. A fourth harmonic (266 nm) of a Nd:YAG laser (Coherent, Brilliant) excited PAHs, and their PLIF signals were detected with an intensified charge-couple device camera (ICCD; Princeton Instruments, PI-MAX2-1024i) with a narrow band-pass filter (central wavelength, $450 \mathrm{~nm}$; full width at half maximum, $25 \mathrm{~nm}$ ). After background noise correction, the PLIF images were normalized with a vertical profile of laser beam (450 $\mu \mathrm{m}$ width and $10 \mathrm{~cm}$ height), which was obtained using a cuvette filled with acetone. Since PAHs have broad absorption and emission characteristics, the measured PLIF signals provided only relative data for comparison purposes. Note that the current detection method adequately represents 2- to 3-ring aromatics [30]. To minimize the 
incandescence signals from soot particles caused by the laser, and to keep the fluorescence emission in a linear regime with respect to the laser energy, the energy density of the laser sheet was kept sufficiently low [31].

An Ar-ion laser (Spectra-Physics, Stabilite 2017), operated at 488 nm, illuminated the seeding $\mathrm{TiO}_{2}$ particles $(\sim 0.2 \mu \mathrm{m})$ to qualitatively visualize the flow patterns of the fuel jets. Due to the layer of beads inside the coflow section, particle seeding to the coflow was not considered. A high-speed camera (Photron MC2) and a digital camera with micro-lenses were used to capture the images.

\section{Results and discussion}

\subsection{Jet velocity effect}

In a previous study [5], the onset condition of ITV formation was elaborated in terms of $V_{\text {ac }}$ and $f_{\text {ac }}$ at fixed propane and coflow velocities, which were chosen as the baseline condition for the present study. We found that $f_{\mathrm{ac}} \times V_{\mathrm{ac}}{ }^{2}$ was reasonably constant at the onset of an ITV. Such a scaled proportionality is consistent with the force acting on charged species due to an induced magnetic field [5]. To explore the effect of flow velocity on the onset of an ITV, the fuel and the coflow velocities were varied (see Table 1 for details). 


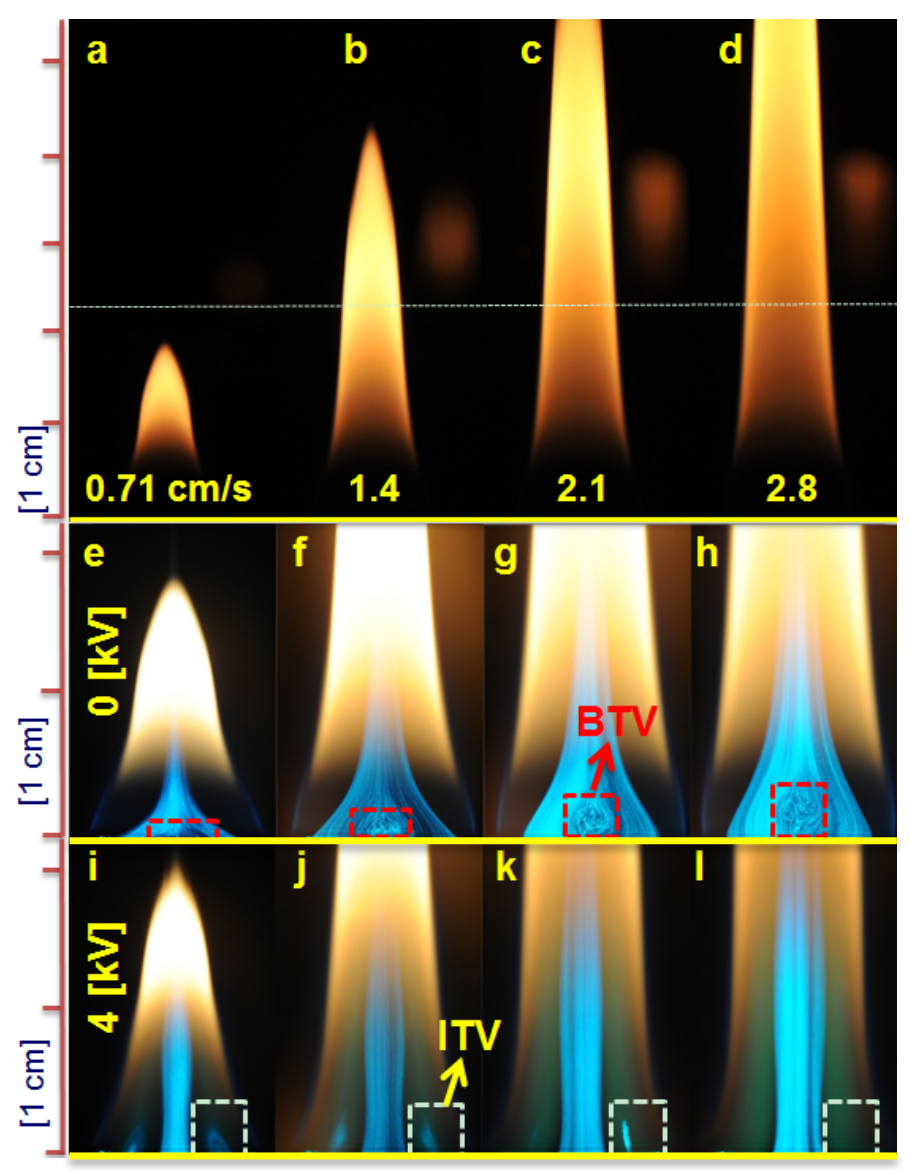

Fig. 2. Flames and their inner flow patterns with various fuel velocities for a fixed coflow velocity at $U_{\mathrm{c}}=6.2$ $\mathrm{cm} / \mathrm{s}$.

Figure 2(a-d) shows photographs (exposure time 1/200 s) of propane flames with the variation in fuel jet velocity $\left(U_{\mathrm{f}}\right)$ at a fixed coflow velocity $\left(U_{\mathrm{c}}=6.2 \mathrm{~cm} / \mathrm{s}\right)$ without applying an electric field, where the baseline condition is (b). The results indicate that the flame (yellow luminous zone) height increases with $U_{\mathrm{f}}$. The horizontal solid yellow line at the bottom of Fig. 2(a-d) represents the location of the nozzle exit. As $U_{\mathrm{f}}$ increases, the height $(H)$ of the yellow luminous zone becomes larger and the dark zone height, defined as the centerline length from the nozzle exit to sooting zone (which is marked as a dotted line for the baseline condition (b) as a reference), becomes longer. The intensity of the soot luminosity near the nozzle becomes weaker, which can be attributed to a reduced 
residence time for soot growth with increased $U_{\mathrm{f}}$.

The near-nozzle flow fields, for the cases without an applied electric field (e-h) (corresponding inner flow structure of $\mathrm{a}-\mathrm{d}$ ), are visualized with the pathlines of the seed particles illuminated by the Ar-ion laser (exposure time $1 / 6 \mathrm{~s}$ ). Note that a recirculation bubble along the central axis of the jet near the nozzle exit was identified as a buoyancy-driven toroidal vortex (BTV, marked as red rectangles). This phenomenon was reported in detail in a previous study [29]. Since propane is heavier than air, it induces a negative (downward) buoyancy to decelerate the velocity of propane fuel, which counteracts the positive (upward) buoyancy of the burnt gas.

When an electric field is applied at $V_{\text {ac }}=4 \mathrm{kV}(\mathrm{i}-1)$ at the onset frequency $f_{\text {ac }}$ of each velocity condition (refer to Fig. 3), the formation of ITVs (marked with yellow squares) were identified. As shown in previous work [5], the Mie scattering signals from the laser illumination in this region can be attributed to fine particles, presumably soot particles, which are trapped in the recirculating structure of the ITV. Note that ITVs cannot be observed by the naked eye because the temperature - which is expected to be higher than ambient due to the recirculating nature - is still not high enough for the soot particles to emit discernable black-body radiation.

As a result of the ITV formation, the effective nozzle area for fuel flow was reduced considerably, therefore a consequent acceleration of fuel in the central region might suppress the formation of a BTV. Another consequence of the increase in local fuel velocity due to the ITV formation was an increase in the flame length (e.g., comparing Fig. 2(e) and Fig. 2(i)) and a decrease in the near-nozzle soot luminosity (Fig. 2(i-1)). Although it is not shown here, a factor of four variation in the coflow velocity $\left(U_{\mathrm{c}}=2.1-\right.$ 
$8.3 \mathrm{~cm} / \mathrm{s}$ ) had a negligible effect on both the flame shape and the ITV formation of the baseline flame. This can be attributed to two factors: (1) the ITV formation typically occurs in the fuel region such that the coflow velocity has little effect, and (2) the buoyancy-induced flow in a diffusion flame is generally stronger (order of $1 \mathrm{~m} / \mathrm{s}$ ) than the coflow velocity tested in this experiment [29].

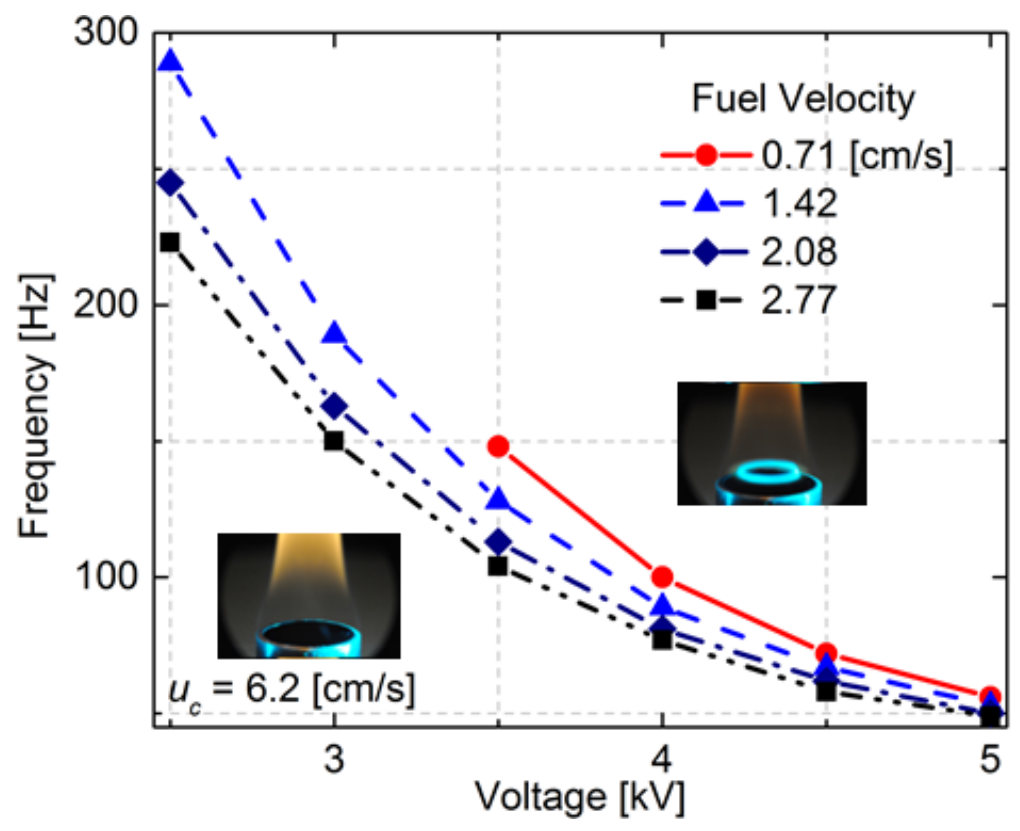

Fig. 3. Onset conditions of the ITV at several fuel velocities for a fixed coflow velocity at $U_{\mathrm{c}}=6.2 \mathrm{~cm} / \mathrm{s}$.

Figure 3 illustrates the onset conditions of ITV formation in terms of $V_{\text {ac }}$ and $f_{\text {ac }}$ under various $U_{\mathrm{f}}$ conditions for a fixed $U_{\mathrm{c}}=6.2 \mathrm{~cm} / \mathrm{s}$. When $U_{\mathrm{f}}=0.71 \mathrm{~cm} / \mathrm{s}$, ITV formation could not be achieved for $V_{\mathrm{ac}}<3.5 \mathrm{kV}$, hence no data in Fig. 3. The inset photos respectively represent a typical inner flow structure in each domain: a normal flame before reaching the onset condition and a flame with the ITV. These were captured using a horizontally-oriented laser illumination for the baseline condition [5]. At a fixed $f_{\text {ac }}$, the onset of the ITV was facilitated at a smaller $V_{\text {ac }}$ as $U_{\mathrm{f}}$ increased. For example, at $f_{\text {ac }}$ $=150 \mathrm{~Hz}$, the ITV was triggered by applying $V_{\mathrm{ac}}=3.5 \mathrm{kV}$ at $U_{\mathrm{f}}=0.71 \mathrm{~cm} / \mathrm{s}$, while only 
$V_{\text {ac }}=3 \mathrm{kV}$ was enough to form the ITV at $U_{\mathrm{f}}=2.8 \mathrm{~cm} / \mathrm{s}$. Similarly, at a fixed $V_{\text {ac }}$, increased $U_{\mathrm{f}}$ resulted in a smaller $f_{\mathrm{ac}}$ for the ITV formation.

An increase in $U_{\mathrm{f}}$ can modify the parameters affecting ITV formation, such as the initial fuel jet momentum and the distribution of local-species concentrations. In the previous study [5], a threshold level of induced magnetic force $\left(\sim f_{\mathrm{ac}} \times V_{\mathrm{ac}}{ }^{2}\right)$, which acts on moving charged particles due to ionic wind, was thought to be required for the ITV formation. In such a case, the electric stimulus should overcome the jet momentum for the bifurcation phenomenon to form an ITV. Thus, it was expected that, as the jet momentum increased ( $U_{\mathrm{f}}$ increased), the induced magnetic force $\left(\sim f_{\mathrm{ac}} \times V_{\mathrm{ac}}{ }^{2}\right)$ should also be higher to trigger the ITV. However, Fig. 3 shows a counterintuitive result: it was easy to form the ITV at a higher fuel jet velocity, with decreased $f_{\mathrm{ac}} \times V_{\mathrm{ac}}{ }^{2}$ at the onset of ITV for higher $U_{\mathrm{f}}$.

Besides increased jet momentum due to increased fuel jet velocity, the outer edge of the fuel path lines (Fig. 2(e-h)), which represented a fuel stream tube, was less deflected toward the center of the jet, implying a narrower fuel-diffusion zone thickness for a higher $U_{\mathrm{f}}$. This might have influenced the profiles of various charged species in this region, in addition to the formation of PAHs and soot particles as charged species that was mentioned previously.

In this regard, a qualitative PLIF measurement of the PAHs was performed for the conditions in Fig. 2. The PLIF images are shown in Fig. 4. The signals were normalized by their respective maximum intensity without applied $V_{\text {ac }}(\mathrm{a}-\mathrm{d})$ and at the moment of ITV formation $(\mathrm{e}-\mathrm{h})$ for $U_{\mathrm{f}}=2.8 \mathrm{~cm} / \mathrm{s}$ and $U_{\mathrm{c}}=6.2 \mathrm{~cm} / \mathrm{s}$. Note that the maximum intensity of the PLIF signal with the ITV was about twofold of that without applied AC. 
For the cases without applied $\mathrm{AC}$, as $U_{\mathrm{f}}$ increased, the intensity of the PAHs increased noticeably in the downstream region. Although this increased concentration of PAHs (since it could be partly interpreted as an increased amount of charged species) might have been related to the early onset of an ITV with increased jet velocity as shown in Fig. 3 , the PAH signals were not very different near the nozzle region where the ITV formed. Once the ITV formed at the onset $f_{\mathrm{ac}}$ for $V_{\mathrm{ac}}=4 \mathrm{kV}(\mathrm{e}-\mathrm{h})$, significantly enhanced PAH signals were observed near the nozzle exit region. This was caused by an increased residence time in the relatively hot recirculation zone of the ITV [5].

So far the experiment with varying fuel jet velocity is inconclusive on the role of PAHs. However, we might hypothesize that the effect of increasing fuel jet velocity on the easier ITV formation can be attributed to producing more charged species in the reaction zone (due to increased supply of fuel), such that increased electric body force near the nozzle rim (due to increased charge density in the flow volume) can facilitate the ITV formation. As previously mentioned, the easier ITV formation with increased jet momentum is counterintuitive and it may not be an aerodynamic effect. Note that the maximum tested jet velocity $(2.8 \mathrm{~cm} / \mathrm{s})$ was very small as compared to buoyancy driven convection [29], which also indicates negligible aerodynamic effect of increased jet momentum for the tested range of velocity. Therefore, when the effects of fuel and the size of nozzle were investigated a careful selection of experimental parameters was required. 


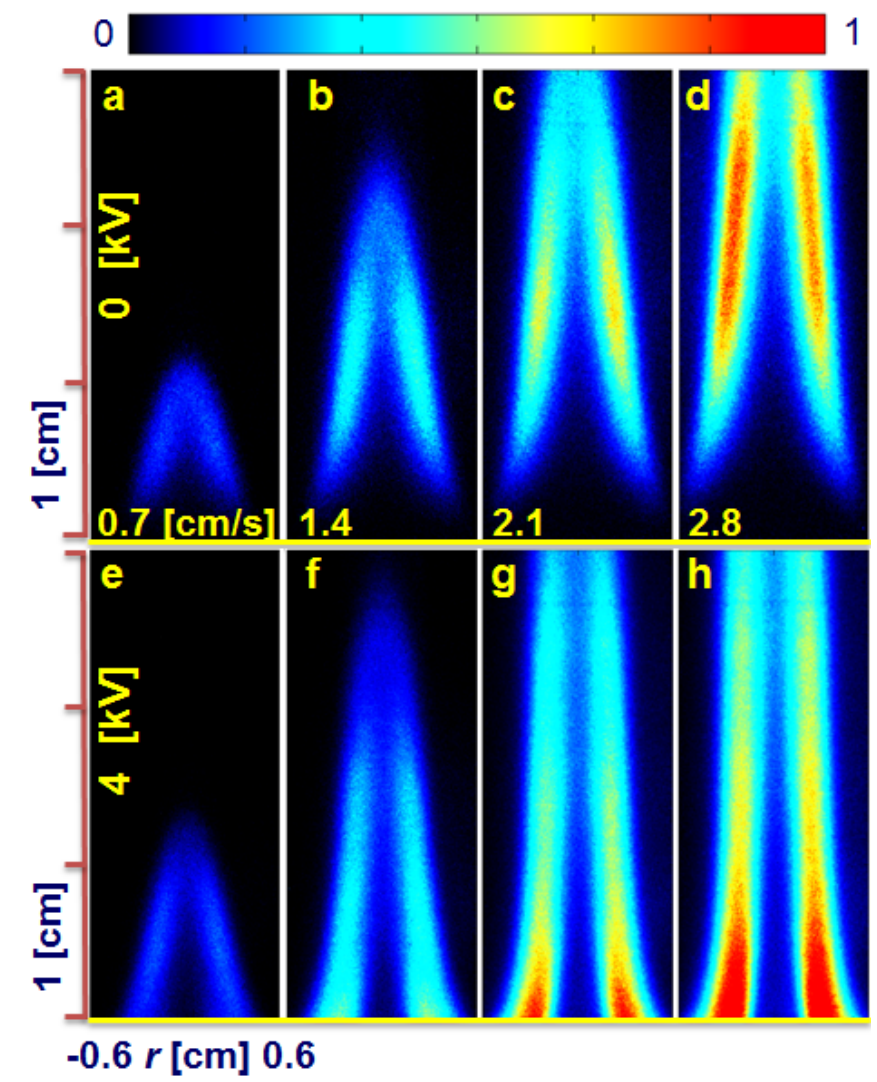

Fig. 4. PLIF images of PAHs with varying fuel velocities for a fixed coflow velocity at $U_{\mathrm{c}}=6.2 \mathrm{~cm} / \mathrm{s}$.

\subsection{Fuel effect on ITV formation}

To evaluate the relative importance of PAHs (especially for ionized PAHs) along with soot on ITV formation, various fuels with different sooting tendencies were considered, including ethylene $\left(\mathrm{C}_{2} \mathrm{H}_{4}\right)$, ethane $\left(\mathrm{C}_{2} \mathrm{H}_{6}\right)$, propane $\left(\mathrm{C}_{3} \mathrm{H}_{8}\right)$, n-butane (n- $\mathrm{C}_{4} \mathrm{H}_{10}$ ), and i-butane $\left(\mathrm{i}-\mathrm{C}_{4} \mathrm{H}_{10}\right)$. The sooting tendency of each fuel, based on the normalized smoke point (NSP) [32], is in the order of: ethylene (NSP 14.6 \pm 8.3 ) > i-butane $(96.3)>$ n-butane $(175 \pm 33) \sim$ propane $(202 \pm 82)>$ ethane $(319 \pm 153)$, which is consistent with the sooting temperature index (STI) determined from counterflow nonpremixed flames [33]. 
Considering that $\mathrm{CH}$ concentration (initiation of chemi-ionization, $\mathrm{CH}+\mathrm{O} \rightarrow$ $\mathrm{CHO}^{+}+\mathrm{e}$ ) would control the total amount of charged species, carbon flux to each flame should be maintained to keep a reasonably same amount of charge generation. However, when we maintained the same carbon flux as that of the reference propane case with other fuels, small variation in flame heights could be found and this might result in inconsistent local electric field intensity at each flame location. To compromise these two factors, the fuel jet velocity was adjusted as $\left.U_{\mathrm{f}}\right|_{\mathrm{C} 2 \mathrm{H} 4}=2.2, U_{\mathrm{f} \mid \mathrm{C} 2 \mathrm{H} 6}=2.2, U_{\mathrm{f} \mid \mathrm{C} 3 \mathrm{H} 8}=1.4$, $U_{\mathrm{f} \mid \mathrm{nC} 4 \mathrm{H} 10}=1.0$, and $U_{\mathrm{f} \mid \mathrm{iC} 4 \mathrm{H} 10}=0.9 \mathrm{~cm} / \mathrm{s}$ at fixed $U_{\mathrm{c}}=6.2 \mathrm{~cm} / \mathrm{s}$ (Table 1$)$, such that each fuel jet exhibited a comparable flame height based on the yellow luminosity. Note that the differences in the carbon flux for $\mathrm{C}_{2} \mathrm{H}_{4}, \mathrm{C}_{2} \mathrm{H}_{6}, \mathrm{n}-\mathrm{C}_{4} \mathrm{H}_{10}$, and $\mathrm{i}-\mathrm{C}_{4} \mathrm{H}_{10}$ as compared to the propane case were $4.8,4.8,-4.8$, and $-14.3 \%$, respectively, showing a reasonable range of variation except i-butane. Based on Fig. 3, $100 \%$ increase in jet velocity $(0.71$ $\mathrm{cm} / \mathrm{s}$ to $1.42 \mathrm{~cm} / \mathrm{s}$ ) roughly decrease the ITV forming AC frequency around $20 \mathrm{~Hz}$ at 3.5 $\mathrm{kV}$, so the effect of $10 \%$ variation in the jet velocity on the ITV formation should be negligible (a few $\mathrm{Hz}$ variation), thus small variation in the carbon flux can be neglected in the following discussion. 


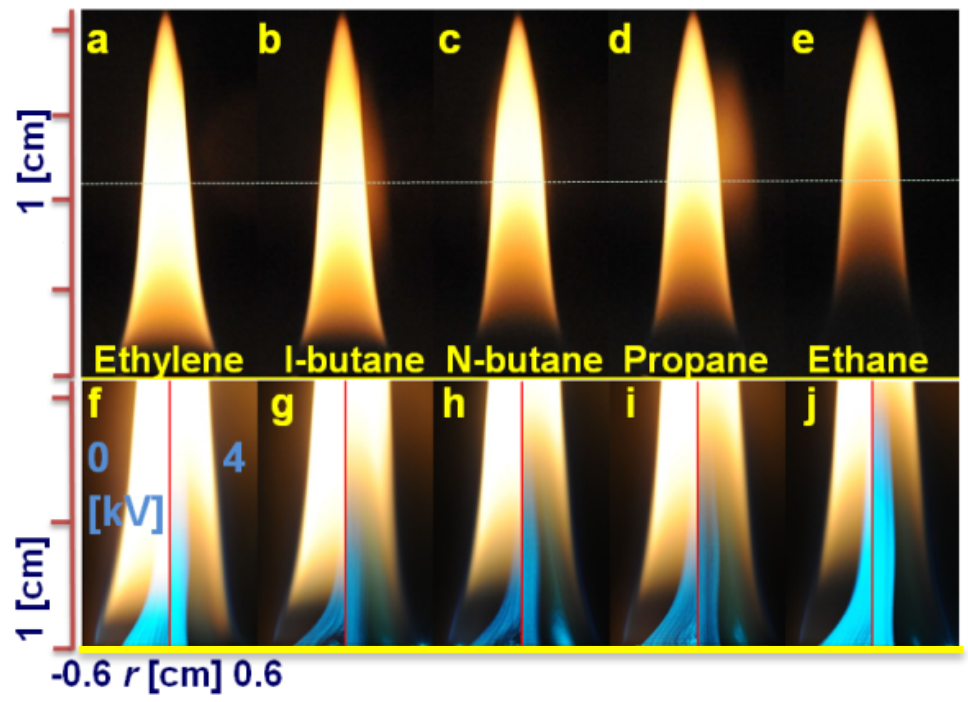

Fig. 5. Flame images and their inner flow patterns with ethylene (a and f), i-butane (b and g), n-butane (c and h), propane ( $d$ and $i)$, and ethane (e and j). The left half images in ( $f-j)$ show fuel streams without applied voltage, while the right ones show fuel streams with $V_{\mathrm{ac}}=4 \mathrm{kV}$; each AC frequency is the onset $f_{\mathrm{ac}}$ indicated in Fig. 6 at $V_{a c}=4 \mathrm{kV}$.

Figure 5(a-e) shows the flame photos of ethylene, i-butane, n-butane, propane, and ethane, respectively, in the order of their intensity of yellow luminosity. The intensity of yellow luminosity and the dark zone height followed the sooting tendency based on NSP. Figure 5(f-j) exhibits close-up photos for the near-field flow pattern with the laser illumination, without (left half) and with (right half) applied voltage $\left(V_{\mathrm{ac}}=4 \mathrm{kV}\right)$. For the cases without applied voltage, the fuels that are heavier than air demonstrated a formation of BTV near the nozzle along the center axis, while neither ethylene nor ethane showed such BTV formation. Without applied voltage, the deflection of the outer edge of the visualized path lines (blue zone) from the seed particles were similar to each other, because the air entrainment induced by buoyancy is reasonably proportional to flame size. For the cases with $V_{\text {ac }}=4 \mathrm{kV}$, similar to Fig. 2(e-1), reduced soot luminosity with an ITV could be observed. Restricted flow area of each fuel stream at the nozzle exit indicated 
the formation of the ITV, although it was not clearly discernible for the ethylene jet. Methane, which is very weakly sooting (no NSP data available in [32]), was also tested; however, the ITV formation could not be achieved within the tested range of AC, and thus it is not shown here.

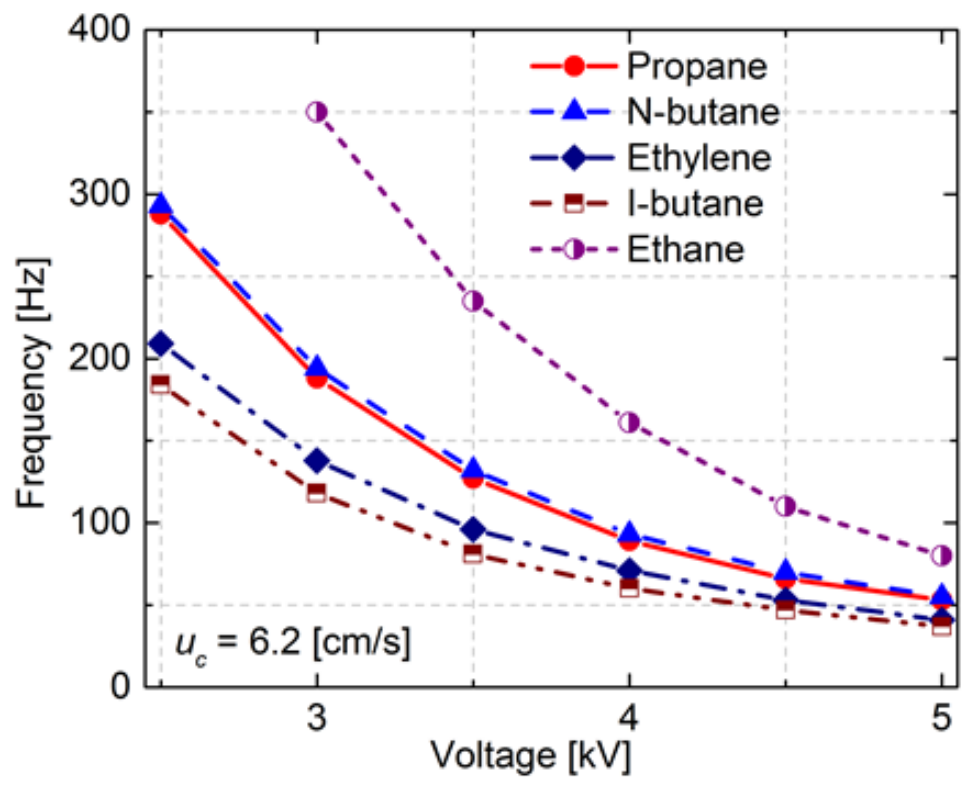

Fig. 6. ITV onset conditions with various fuels.

Figure 6 shows the onset conditions of an ITV for the tested fuels in terms of $V_{\text {ac }}$ and $f_{\text {ac }}$. When a tendency of ITV formation is introduced, it is in the order of i-butane $>$ ethylene $>$ propane $\sim$ n-butane $>$ ethane $>>$ methane, e.g., at $f_{\text {ac }}=150 \mathrm{~Hz}$, required $V_{\text {ac }}$ are 2.8 (i-butane), 3.0 (ethylene), 3.3 (propane and n-butane), and $4.2 \mathrm{kV}$ (ethane). Since the most discernable factor between the various fuels is the sooting tendency, a comparison between the tendency of ITV formation and the sooting tendency might suggest an important clue as to ITV formation. However, the results show an inconsistency between these two factors, particularly for ethylene and i-butane. The i-butane flame was less sooting than ethylene, while the ITV was more easily triggered for i-butane than ethylene. 
This result implies that thermally-ionized nascent soot particles may not play a dominant role in ITV formation.

To further understand the reason behind such a high tendency of ITV formation with i-butane, the PAH PLIF images were recorded; the results are illustrated in Fig. 7. For each fuel, the left-half image was taken without applied voltage and the right-half image was captured with $V_{\text {ac }}=4 \mathrm{kV}$, with the corresponding onset $f_{\text {ac }}$ shown in Fig. 6 . Stronger intensity of PAHs in the near-nozzle region with an ITV was observed for each fuel for the same reason explained previously.

The maximum strength of each PAH PLIF signal intensity is in the order of i-butane $>$ n-butane $\sim$ propane $>$ ethylene $>$ ethane. This differs from the order of ITV tendency, which is how Fig. 7 was arranged. Two points should be noted from the results in Fig. 7: (1) although ethylene showed the highest sooting tendency, its PAH intensity was even smaller than propane's, while the PAH intensity with i-butane was the strongest, showing a factor of difference compared to other fuels, and (2) an early formation of PAHs (similar in size to the PAHs detected in the present setup) was found in ethylene and i-butane as compared to other fuels, which resulted in the existence of PAHs closer to the nozzle exit. Concerning the weak PAH PLIF signal of ethylene flame, it was previously reported $[34,35]$ and later substantiated via simulations [36] that, although the soot production in ethylene flame is higher than that in propane flame, the production of PAHs is higher for propane than for ethylene in counterflow nonpremixed flame experiments. 


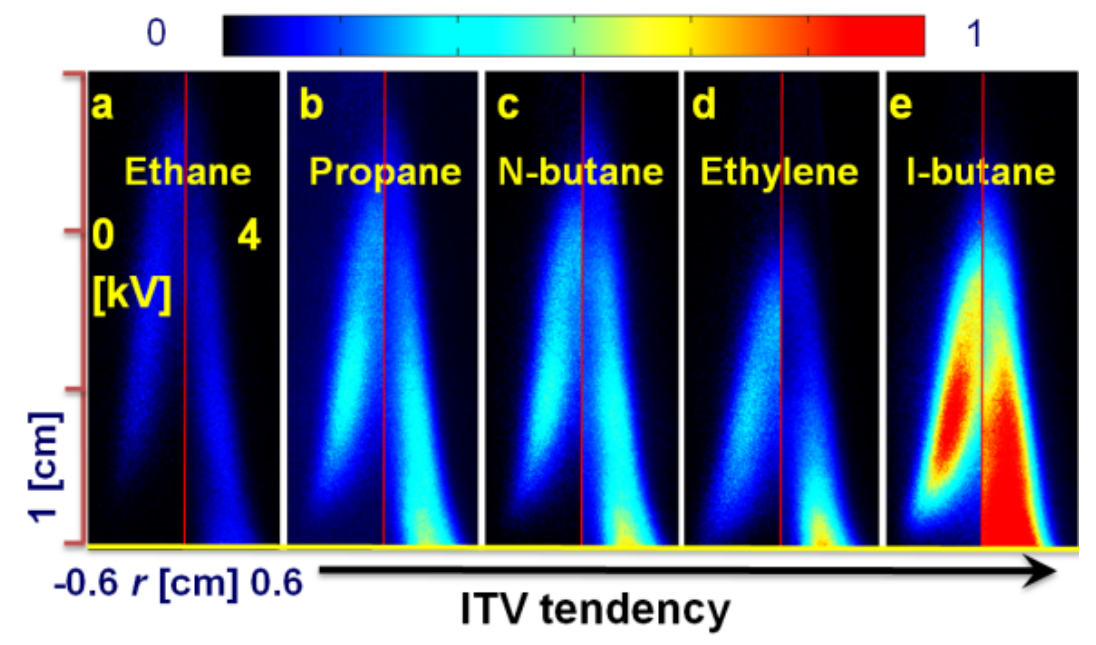

Fig. 7. PAH PLIF images are shown for ethane (a), propane (b), n-butane (c), ethylene (d), and i-butane (e). Left half images in (a-e) show PAHs without applied voltage, while the right ones with $V_{a c}=4 \mathrm{kV}$; each AC frequency is the onset $f_{\mathrm{ac}}$ indicated in Fig. 6 at $V_{\mathrm{ac}}=4 \mathrm{kV}$.

If one assumes that ionized PAHs are the most influential ionized species for ITV formation, then the highest tendency of ITV formation with i-butane can be readily explained due to its strong PAH signal. Because the local electric field intensity becomes stronger as the distance from the nozzle rim becomes smaller, the existence of PAHs closer to the nozzle exit with ethylene may play an important role in the higher ITV tendency of ethylene than of propane or n-butane. Thus, it may be concluded that ionized PAHs are a key species for the ITV formation, and that the spatial distribution and concentration of PAHs are equally important in the present jet configuration, due to a non-uniform electric field (particularly, greater field intensity closer to the nozzle rim).

\subsection{Nozzle size effect}

In the section above, the importance of ionized PAHs on ITV formation was demonstrated. Since ITV formation could also be related to a geometrical configuration, 
various nozzle sizes were tested on the tendency of ITV formation (as shown in Table 1). Figure 8 shows the photographs of propane flames for five different nozzle diameters as $d$ $=2.8,4.5,7.6,10.8$, and $12.5 \mathrm{~mm}$. Again, uniform flame heights were maintained by adjusting the jet velocity of propane at $U_{\mathrm{f}}=18.3,7.5,2.7,1.4$, and $1.0 \mathrm{~cm} / \mathrm{s}$ for $d=2.8$, 4.5, 7.6, 10.8, and $12.5 \mathrm{~mm}$, respectively. The resulted differences in the carbon flux for $d$ $=2.8,4.5,7.6$, and 12.5 were $-12.1 \%,-7.0 \%,-4.5 \%$, and $-4.3 \%$, respectively as compared to the reference case of $d=10.8 \mathrm{~mm}$, thus a velocity effect to correct the carbon flux should be minimal to the overall trend.

Regardless of the nozzle diameter, the yellow luminosity remained similar in height and intensity, and similar dark zone heights near the nozzle exit could be observed. One visible difference was the flame diameter, particularly near the nozzle rims, due to a flow restriction caused by the nozzle size.

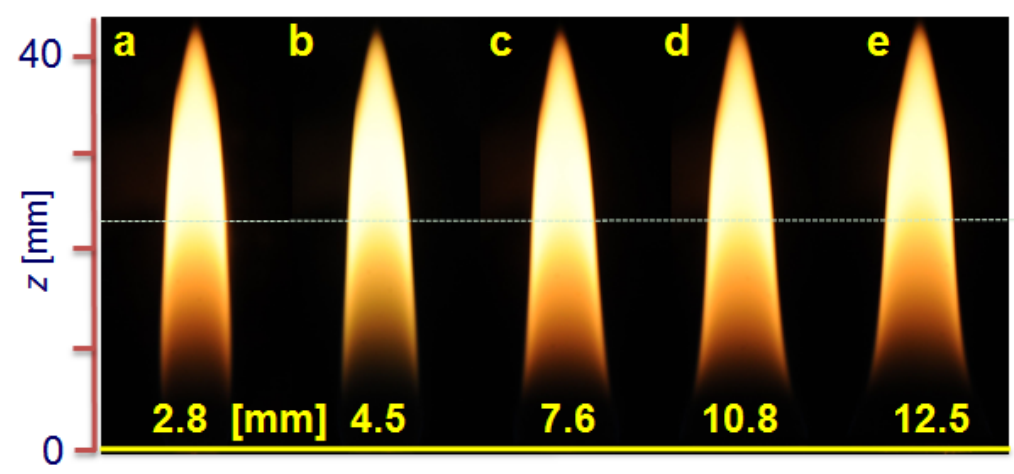

Fig. 8. Flame photos with various nozzle diameters. Jet velocities are $U_{\mathrm{f}}=18.3$ (a), 7.5 (b), 2.7 (c), 1.4 (d), and $1.0(e) \mathrm{cm} / \mathrm{s}$.

Comparisons of near-nozzle flow structures before and after the formation of an ITV (4 kV with the corresponding onset $f_{\mathrm{ac}}$ in Fig. 10), are illustrated in Fig. 9(a-e). Except for the smallest $d$ tested $(2.8 \mathrm{~mm})$, the formation of an ITV could be clearly identified, indicating a contraction of the fuel streams due to the ITV formation as shown 
in Fig. 9(b-e). The size of the ITV increased as the nozzle diameter increased. However, for $d=2.8 \mathrm{~mm}$ (Fig. 9(a)), a clearly observable ITV flow pattern could not be identified. Instead, as shown in Fig. 9(a), thin and straight pathlines for the fuel stream were totally destroyed with the electric field, exhibiting unsteady particle motion in the middle, around $3 \mathrm{~mm}$ above the nozzle (Supplementary Movie 1: $V_{\mathrm{ac}}=4 \mathrm{kV}$ and $f_{\mathrm{ac}}=63 \mathrm{~Hz}$ ).

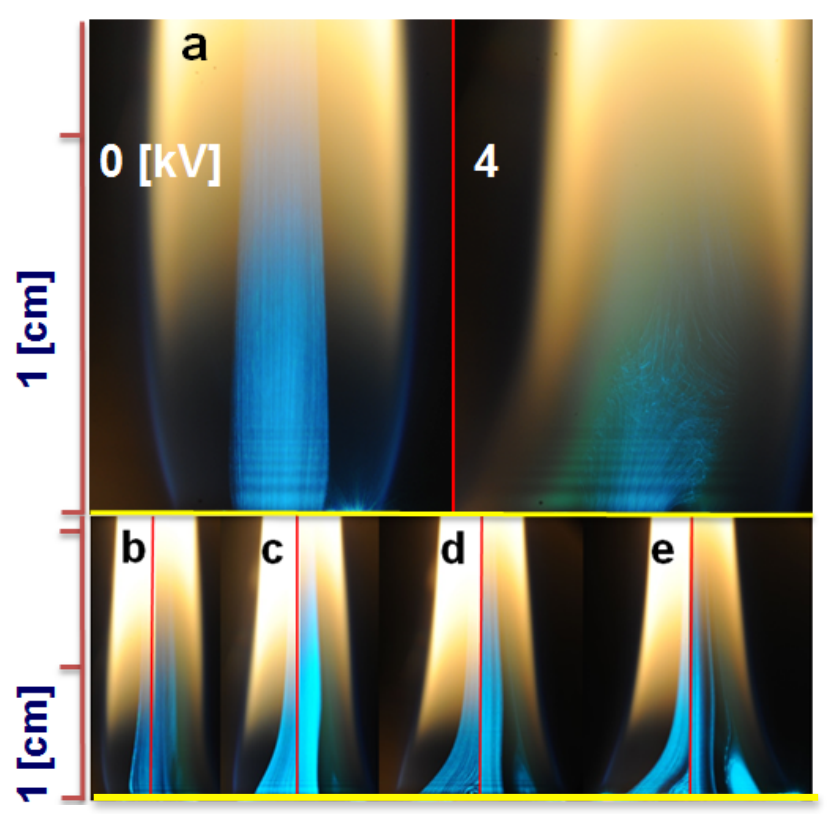

Fig. 9. Near-nozzle flow patterns with various nozzle diameters, where (a-e) correspond to the flames in Fig. 8. Left half images in (b-e) show flow pattern without applied voltage, while the right ones with $V_{\mathrm{ac}}=4 \mathrm{kV}$; each $\mathrm{AC}$ frequency is the onset $f_{\mathrm{ac}}$ indicated in Fig. 10 at $V_{\mathrm{ac}}=4 \mathrm{kV}$.

To detail this unsteady flow pattern with $d=2.8 \mathrm{~mm}$, the seeding particle motions in Fig. 9(a) at $V_{\text {ac }}=4 \mathrm{kV}$ (after ITV formation) were investigated using the high-speed camera at $250 \mathrm{fps}$. In Supplementary Movie 2, a periodically-produced flow structure due to $\mathrm{AC}$ was identified - it destroyed a fuel core located about $3 \mathrm{~mm}$ downstream. The frequency of this unsteady structure was 63 , the same as $f_{a c}$, indicating the essential role 
of AC-driven instability. As $V_{\text {ac }}$ became smaller, such as $2.5 \mathrm{kV}$, the onset frequency increased to $198 \mathrm{~Hz}$ (Fig. 10). With this faster AC frequency, a rather steady ITV, similar to that shown in Fig. 9(b), was also found for the $2.8 \mathrm{~mm}$ nozzle. In Fig. 10, the definition of the onset condition for this smallest nozzle refers to the onset of either the steady ITV or the unsteady destruction of a fuel stream. Note that for larger-sized nozzles (such as the $10.8 \mathrm{~mm}$ nozzle), the destruction of a fuel core could not be observed within the tested range of AC frequency. This indicates the importance of length scales, including both the nozzle diameter and the size of the ITV, in obtaining a steady, balanced flow field.

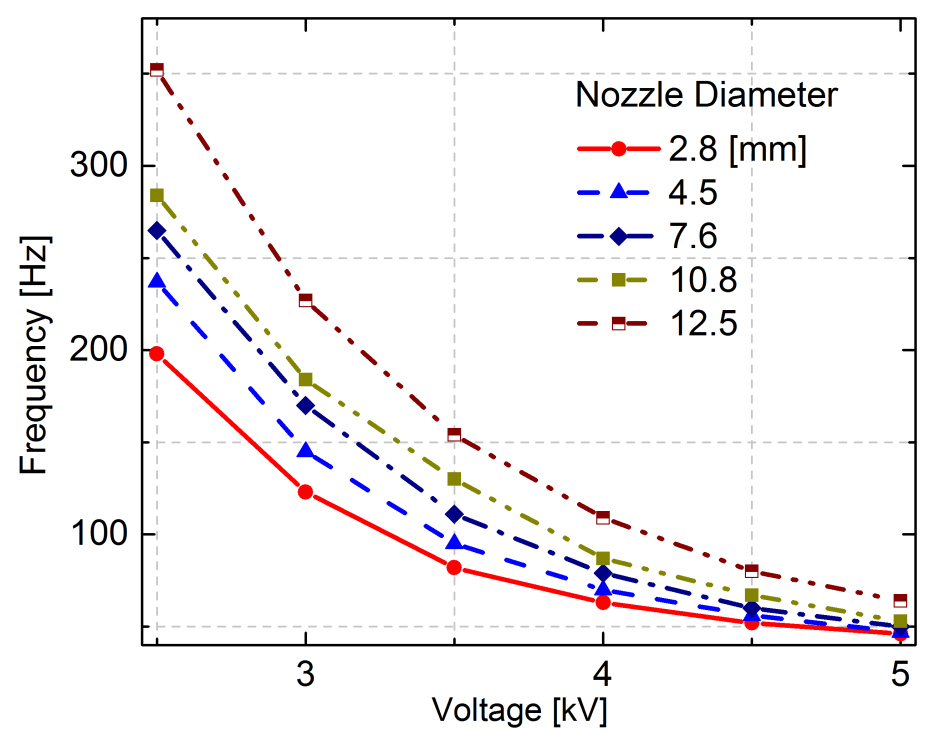

Fig. 10. ITV onset conditions with various nozzle diameters.

Figure 10 illustrates the onset conditions of ITV formation in terms of $V_{\mathrm{ac}}$ and $f_{\mathrm{ac}}$ for various nozzle diameters. The formation of the ITV (or the modification of fuel stream for the case of $d=2.8 \mathrm{~mm}$ ) became easier as the nozzle diameter decreased, 
indicating that a smaller $V_{\mathrm{ac}}$ is sufficient to generate an ITV (or destroy a fuel core), with a smaller $d$ for the same $f_{\text {ac. }}$ This again implies that jet momentum is not the key parameter in controlling the formation of ITVs.

To explore the reason behind this observation, we analyzed the result of $\mathrm{PAH}$ PLIF signals for selected nozzle diameters $(4.5,10.8$ and $12.5 \mathrm{~mm}$, shown in Fig. 11), without and with an electric field ( $V_{\mathrm{ac}}=4 \mathrm{kV}$ with the corresponding onset $f_{a c}$ in Fig. 10). Similar PAH distributions were found with reasonably similar peak concentrations. As $d$ became larger, the width of PAH distribution at the bottom of the flames increased and the concentration at the top near the center decreased, indicating no clear clue as to the ITV tendency shown in Fig. 10. The relative location of PAHs to the nozzle rim is illustrated schematically in Fig. 12. For a large size nozzle, the PAHs moved away from the nozzle rim, toward the center (e.g. $d=12.5 \mathrm{~mm}$ ). As $d$ became smaller (for example $d$ $=4.5 \mathrm{~mm}$ ), the bottom-edge of PAHs migrated radially outside (basically the nozzle rim repositioned inwardly relative to the PAHs). As a result, the PAHs settled just above the nozzle rim. Thus, a shorter distance between the PAH zone and the nozzle rim resulted in increased local electric field intensity for smaller nozzles [37]. A further decrease in the nozzle diameter $(d=2.8 \mathrm{~mm})$ may lead to the destruction of a fuel core at high voltage, low frequency conditions, indicating a different interaction between the ionic wind and the preexisting intrinsic flow patterns of a diffusion flame. 


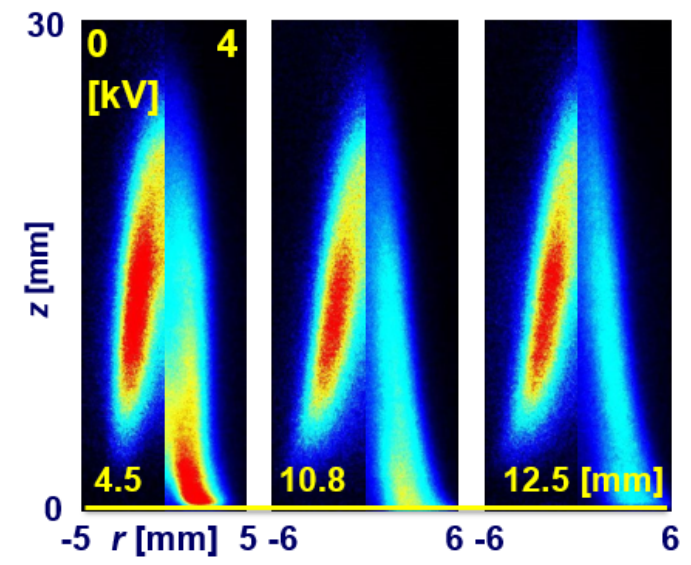

Fig. 11. PLIF images of PAHs with various nozzle diameters. The left-half images show PAHs without applied voltage, while the right-half images illustrate PAHs with applied voltage $V_{a c}=4 \mathrm{kV}$; each AC frequency is the onset $f_{\mathrm{ac}}$ indicated in Fig. 10 at $V_{\mathrm{ac}}=4 \mathrm{kV}$.

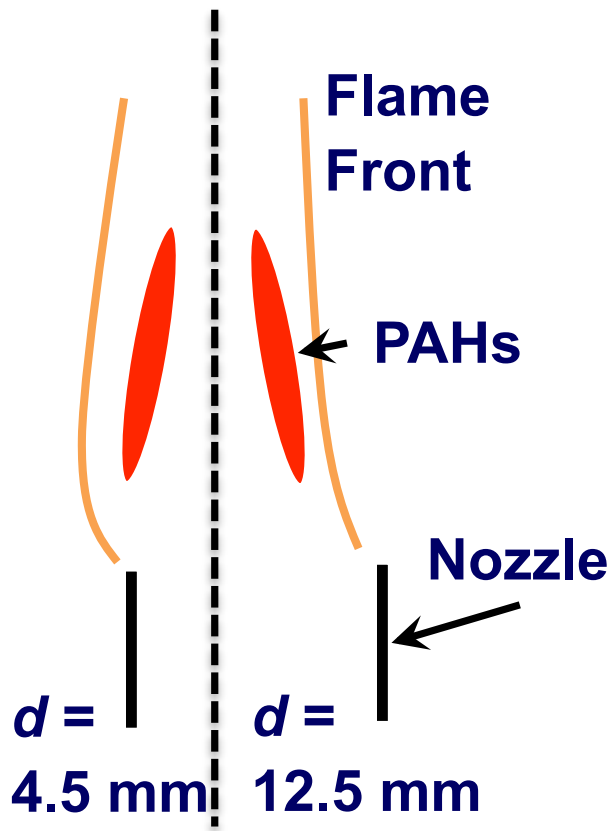

Fig. 12. Schematic of the relative location of PAHs and nozzle rims.

\section{Conclusions}

A detailed, experimental parametric study was conducted to elaborate on the onset conditions of an electro-magnetically-induced inner toroidal vortex in small, jet diffusion 
flames by applying AC to the nozzles. By changing fuel and coflow velocities, it was found that mild fluid dynamic effects on the ITV formation should be minimal, and that increased concentrations of PAHs should have a primary impact, because the ITV was easily triggered with a higher fuel velocity, yielding more PAHs.

Hypothesizing that a portion of ionized PAHs should be maintained, various fuels with different sooting tendencies were tested. It was determined that the ITV tendency was not related to the sooting tendency, illustrating the minor role of nascent-charged soot particles in forming an ITV. However, the ITV tendency can be qualitatively well correlated with a combination of PAH concentrations and their relative locations from a nozzle rim, because the intensity of the local electric field at the location of PAHs is an important factor in determining the Lorenz force acting on ionized PAHs. By using nozzles of many different sizes, we concluded that the relative distance between the nozzle rim and the PAHs clearly plays a crucial role in the ITV formation. We also discovered that below a certain nozzle size, an unsteady interaction between the AC-driven flow structure and a fuel stream could be produced.

\section{Acknowledgments}

The research reported in this publication was supported by Competitive Research Funding from King Abdullah University of Science and Technology (KAUST).

\section{References}


[1] S.H. Won, S.K. Ryu, M.K. Kim, M.S. Cha, S.H. Chung, Effect of electric fields on the propagation speed of tribrachial flames in coflow jets, Combust. Flame 152 (2008) 496-506.

[2] J.D.B.J. van den Boom, A.A. Konnov, A.M.H.H. Verhasselt, V.N. Kornilov, L.P.H. de Goey, H. Nijmeijer, The effect of a DC electric field on the laminar burning velocity of premixed methane/air flames, Proc. Combust. Inst. 32 (2009) 1237-1244.

[3] M.S. Cha, Y. Lee, Premixed combustion under electric field in a constant volume chamber, IEEE Trans. Plasma Sci. 40 (2012) 3131-3138.

[4] F. Altendorfner, J. Kuhl, L. Zigan, A. Leipertz, Study of the influence of electric fields on flames using planar LIF and PIV techniques. Proc. Combust. Inst. 33 (2011) 3195-3201.

[5] Y. Xiong, M.S. Cha, S.H. Chung, AC electric field induced vortex in laminar coflow diffusion flames, Proc. Combust. Inst. 35 (2015) 3513-3520.

[6] M.-V. Tran and M.S. Cha, Propagating nonpremixed edge-flames in a counterflow, annular slot burner under DC electric fields, Combust. Flame 173 (2016) 114-122.

[7] M.-V. Tran and M.S. Cha, Time evolution of propagating nonpremixed flames in a counterflow, annular slot burner under AC electric fields, Proc. Combust. Inst. 36, 2017, 1421-1430.

[8] A.M. Drews, L. Cademartiri, M.L. Chemama, M.P. Brenner, G.M. Whitesides, K.J.M. Bishop, AC electric fields drive steady flows in flames, Phys. Rev. E 86 (2012) 036314.

[9] Y. Xiong, S.H. Chung, and M.S. Cha, Instability and electrical response of small laminar coflow diffusion flames under AC electric fields: Toroidal vortex formation and oscillating and spinning flames, Proc. Combust. Inst. 36, 2017, 1621-1628. 
[10] M.K. Kim, S.H. Chung, H.H. Kim, Effect of electric fields on the stabilization of premixed laminar bunsen flames at low AC frequency: Bi-ionic wind effect, Combust. Flame 159 (2012) 1151-1159.

[11] M.K. Kim, S.K. Ryu, S.H. Won, S.H. Chung, Electric fields effect on liftoff and blowoff of nonpremixed laminar jet flames in a coflow, Combust. Flame 157 (2010) $17-24$.

[12] S.M. Lee, C.S. Park, M.S. Cha, S.H. Chung, Effect of electric fields on the liftoff of nonpremixed turbulent jet flames, IEEE Trans. Plasma Sci. 33 (2005) 1703-1709.

[13] D.A. Lacoste, Y. Xiong, J.P. Moeck, S.H. Chung, W.L. Roberts, and M.S. Cha, Transfer functions of laminar premixed flames subjected to forcing by acoustic waves, AC electric fields, and non-thermal plasma discharges, Proc. Combust. Inst. 36, 2017, 4183-4192.

[14] A.P. Chattock, On the velocity and mass of the ions in the electric wind in air, Philos. Mag. 48 (1899) 401-420.

[15] E.N. Volkov, V.N. Kornilov, L.P.H. de Goey, Experimental evaluation of DC electric field effect on the thermoacoustic behaviour of flat premixed flames, Proc. Combust. Inst. 34 (2013) 955-962.

[16] S.D. Marcum, B.N. Ganguly, Electric-field-induced flame speed modification, Combust. Flame 143 (2005) 27-36.

[17] E. Pfender, Thermal plasma technology: where do we stand and where are we going? Plasma Chem. Plasma Process. 19 (1999) 1-31.

[18] J. Kuhl, G. Jovicic, L. Zigan, S. Will, A. Leipertz, Influence of electric fields on premixed laminar flames: Visualization of perturbations and potential for suppression of thermoacoustic oscillations, Proc. Combust. Inst. 35 (2015) 3521-3528.

[19] S. Karnani, D. Dunn-Rankin, F. Takahashi, Z.G. Yuan, D. Stocker, Simulating 
gravity in microgravity combustion using electric fields, Combust. Sci. Technol. 184 (2012) 1891-1902.

[20] M.J. Papac, D. Dunn-Rankin, Modelling electric field driven convection in small combustion plasmas and surrounding gases, Combust. Theor. Model. 12 (2007) $23-44$.

[21] A.B.S. Alquaity, B. Chen, J. Han, H. Selim, M. Belhi, Y. Karakaya, T. Kasper, S.M. Sarathy, F. Bisetti, A. Farooq, New insights into methane-oxygen ion chemistry, Proc. Combust. Inst. 36 (2017) 1213-1221.

[22] N. Speelman, M. Kiefer, D. Markus, U. Maas, L.P.H. de Goey, J.A. van Oijen, Validation of a novel numerical model for the electric currents in burner-stabilized methane-air flames, Proc. Combust. Inst. 35 (2015) 847-854.

[23] J. Han, M. Belhi, T.A. Casey, F. Bisetti, H.G. Im, J.-Y. Chen, The i-V curve characteristics of burner-stabilized premixed flames: detailed and reduced models, Proc. Combust. Inst. 36 (2017) 1241-1250.

[24] H.F. Calcote, Ion production and recombination in flames, Symp. (Int.) Combust. 8 (1961) 184-199.

[25] J.M. Goodings, D.K. Bohme, C.W. Ng, Detailed ion chemistry in methane-oxygen flames. I. Positive ions, Combust. Flame 36 (1979) 27-43.

[26] J.M. Goodings, D.K. Bohme, C.W. Ng, Detailed ion chemistry in methane-oxygen flames. II. Negative ions, Combust. Flame 36 (1979) 45-62.

[27] L. Delfau, P. Michaud, A. Barassin, Formation of small and large positive ions in rich and sooting low-pressure ethylene and acetylene premixed flames, Combust. Sci. Technol., 20 (1979) 165-177. 
[28] A.B. Fialkov, Investigations on ions in flames, Prog. Energy Combust. Sci. 23 (1997) 399-528.

[29] Y. Xiong, M.S. Cha, S.H. Chung, Fuel density effect on near nozzle flow field in small laminar coflow diffusion flames, Proc. Combust. Inst. 35 (2015) 873-880.

[30] K. Kohse-Höinghaus, J.B. Jeffries, Applied combustion diagnostics, Taylor and Francis, New York-London, 2002.

[31] R.K. Hanson, R.M. Spearrin, C.S. Goldenstein, Spectroscopy and optical diagnostics for gases, Springer, 2016.

[32] L. Li, P.B. Sunderland, An improved method of smoke point normalization, Combust. Sci. Technol. 184 (2012) 829-841.

[33] Y. Wang, S.H. Chung, Effect of strain rate on sooting limits in counterflow diffusion flames of gaseous hydrocarbon fuels: Sooting temperature index and sooting sensitivity index, Combust. Flame 161 (2014) 1224-1234.

[34] J.Y. Hwang, W. Lee, H.G. Kang, S.H. Chung, Synergistic effect of ethylenepropane mixture on soot formation in laminar diffusion flames, Combust. Flame 114 (1998) 370-380.

[35] S.S. Yoon, S.M. Lee, and S.H. Chung, Effect of mixing methane, ethane, propane, and propene on the synergistic effect of PAH and soot formation in ethylene-base counterflow diffusion flames, Proc. Combust. Inst. 30 (2005) 1417-1424.

[36] Y. Wang, A. Raj, S.H. Chung, A PAH growth mechanism and synergistic effect on PAH formation in counterflow diffusion flames, Combust. Flame 160 (2013) 1667-1676.

[37] Y. Xiong, D.G. Park, B.J. Lee, S.H. Chung, M.S. Cha, DC field response of one-dimensional flames using an ionized layer model, Combust. Flame 163 (2016) 317-325. 


\section{Supplementary Movies}

Supplementary Movie 1. Near-nozzle flow structures illuminated via Ar-ion laser at $V_{\text {ac }}$ $=4 \mathrm{kV}$ and $f_{\mathrm{ac}}=63 \mathrm{~Hz}$ with 50 fps.

Supplementary Movie 2. Near-nozzle flow structures illuminated via Ar-ion laser at $V_{\text {ac }}$ $=4 \mathrm{kV}$ and $f_{\mathrm{ac}}=63 \mathrm{~Hz}$ with 250 fps. 